OPEN ACCESS

Edited by:

Geoffrey Meru,

University of Florida, United States

Reviewed by:

Zhang Yuehuan,

South China Sea Institute

of Oceanology, China

Jaap Van Tuyl,

Wageningen University \& Research,

Netherlands

Jinhu Wu,

The New Zealand Institute for Plant and Food Research Ltd.,

New Zealand

Wen-Wu Guo,

Huazhong Agricultural University,

China

*Correspondence:

Pingdong Zhang

zhangpd@bjfu.edu.cn

Specialty section:

This article was submitted to Plant Breeding,

a section of the journal

Frontiers in Plant Science

Received: 21 October 2019

Accepted: 27 February 2020

Published: 17 March 2020

Citation:

Zhou Q, Wu J, Sang Y, Zhao Z, Zhang P and Liu M (2020) Effects of Colchicine on Populus canescens

Ectexine Structure and $2 n$ Pollen Production. Front. Plant Sci. 11:295.

doi: 10.3389/fpls.2020.00295

\section{Effects of Colchicine on Populus canescens Ectexine Structure and 2n Pollen Production}

\author{
Qing Zhou ${ }^{1,2,3,4}$, Jian Wu 1,2,3,4, Yaru Sang $^{1,2,3,4}$, Zhengyang Zhao ${ }^{3}$, Pingdong Zhang ${ }^{1,2,3,4 *}$ \\ and Meiqin Liu' ${ }^{1,2}$ \\ ${ }^{1}$ Beijing Advanced Innovation Center for Tree Breeding by Molecular Design, Beijing Forestry University, Beijing, China, \\ ${ }^{2}$ National Engineering Laboratory for Tree Breeding, Beijing Forestry University, Beijing, China, ${ }^{3}$ Key Laboratory of Genetics \\ and Breeding in Forest Trees and Ornamental Plants, Ministry of Education, Beijing Forestry University, Beijing, China, \\ ${ }^{4}$ School of Bioscience and Biotechnology, Beijing Forestry University, Beijing, China
}

Triploid breeding is a central way to improve growth traits, timber quality, and stress resistance in Populus. In the present study, the morphology and viability of colchicineinduced $2 \mathrm{n}$ pollen, triploid production by crossing induced $2 \mathrm{n}$ pollen, and identification of genetic constitution of colchicine-induced $2 n$ pollen were conducted in Populus canescens based on optimizing technology for inducing chromosome doubling in pollen. We found that the meiotic stage, injection time, and the interaction between the meiotic stage and injection time had highly significant effects on the $2 \mathrm{n}$ pollen production rate. The most effective treatment for inducing $2 \mathrm{n}$ pollen was to give 11 injections of $0.5 \%$ colchicine solution when pollen mother cells (PMCs) were at the pachytene stage. The highest $2 \mathrm{n}$ pollen production rate was $30.27 \pm 8.69 \%$. Colchicine occasionally affected ectexine deposition, and some narrow furrows were detected in the ectexine structure. However, no significant difference was observed in the pollen germination rate between natural $2 \mathrm{n}$ pollen and colchicine-induced $2 \mathrm{n}$ pollen. Moreover, 5 triploids derived from FDR-type $2 n$ pollen were generated by crossing induced $2 \mathrm{n}$ pollen, suggesting that colchicine does not eliminate the function of colchicine-induced $2 n$ pollen. However, slower growth of $2 n$ pollen tubes was responsible for a lower triploid production rate.

Keywords: Populus canescens, colchicine, chromosome doubling, $2 \mathrm{n}$ pollen viability, triploid

\section{INTRODUCTION}

Populus canescens (section Populus, family Salicaceae, genus Populus) grows in the Irtysh River Basin, Xinjiang Uygur Autonomous Region, in northwest China. Due to its high ornamental value, resistance to disease, insects, and drought, it is widely used for ecological protection in northwest China (Xu, 2008). However, the annual growth rate of P. canescens is lower owing to its shorter growing season. A long-term breeding program was developed by Beijing Forestry University to improve P. canescens growth. In 1989, a cross-breed between Populus tomentosa $\times$ Populus bolleana and $P$. canescens was first reported by $\mathrm{Li}$ and Zhu (1989), and six superior hybrid clones were developed. In a subsequent study, Duan and Yang (1998) evaluated the cold and drought resistance of these superior hybrids by the anatomical structure of the leaves, water loss rate through the 
leaves, and electroconductivity of the shoots. Tian J. et al., 2018 systematically studied the abnormal behavior of chromosomes during meiosis of pollen mother cells (PMCs), pollen morphology and pollen abortion in some male hybrids between $P$. tomentosa $\times P$. bolleana and $P$. canescens.

Triploid breeding is one of most powerful approaches to improve the genus Populus. The first discovered triploid $(2 \mathrm{n}=3 \mathrm{x}=57)$ individual Populus was a giant form of Populus tremula from a natural population in Sweden, which was characterized by extremely large leaves and rapid growth (Nilsson-Ehle, 1936; Müntzing, 1936). Zhu et al. (1998) reported that genetic gains in the stem volume of 9-year-old natural P. tomentosa triploid clones was higher (56.4\%) than that of the diploid counterparts. In another study, Zhu et al. (1995) documented that the stem volume of the allotriploid clone B301 was 3.5 times higher that of $P$. tomentosa diploids. Subsequently, Zhang et al. (2014a) reported that the fiber length of triploid white poplar hybrid clones was $20.6 \%$ larger than that of diploids. The holocellulose of triploid white poplar hybrid clones was higher $1.3 \%$ than that of its diploid counterparts. However, the lignin content of triploid white poplar hybrid clones was shown to be $21.7 \%$ lower than that of diploid individuals (Zhang et al., 2014b). Therefore, triploid breeding has not only improved the growth rate but also the wood quality of Populus (Zhang et al., 2012, 2013, 2015a,b).

Few studies have focused on $P$. canescens triploid breeding. Only Tian M. et al. (2018) reported $2 \mathrm{n}$ pollen production induced by high-temperature exposure and 42 triploids were obtained. Subsequently, the $2 \mathrm{n}$ pollen $P$. canescens high-temperatureinduced was demonstrated to have originated from second division restitution (SDR) during the meiosis of PMCs. The lower heterozygosity transmitted from the male parent provides SDR-type $2 \mathrm{n}$ gametes a lower breeding value than first division restitution (FDR)-type 2n gametes (Veilleux, 1985; Dong et al., 2015). Thus, crossing FDR-type 2 n gametes with haploid gametes to create $P$. canescens triploids is a promising way to increase growth rate.

The objective of this study was to produce FDR-type $2 n$ pollen by colchicine induction and generate a triploid $P$. canescens germplasm. To optimize the pollen chromosome doubling technology to produce $2 \mathrm{n}$ pollen using colchicine, pollen morphology, $2 \mathrm{n}$ pollen germination in vitro, and genetic constitution identification were conducted, followed by triploid production using colchicine-induced $2 \mathrm{n}$ pollen. Our findings will lay the foundation for the triploid breeding of $P$. canescens.

\section{MATERIALS AND METHODS}

\section{Plant Materials}

Populus canescens and Populus hopeiensis are native tree species in northwestern China. Populus alba $\times$ Populus glandulosa, a hybrid with good fertility under normal conditions, was introduced from Korea in 1984. Male floral branches of P. canescens "YHY1" were collected from a natural population in Aletai, Xinjiang Uygur Autonomous Region. Female branches of P. hopeiensis "HBY1" were collected from the Baotou,
Inner Mongolia Autonomous Region, and female branches of $P$. alba $\times$ P. glandulosa "YXY1" were collected from the Guan Country Nursery, Shandong Province. All sampled floral branches were trimmed and cultured in tap water at the Beijing Forestry University greenhouse $\left(10-20^{\circ} \mathrm{C}\right)$. No additional nutrition was added.

\section{2n Pollen Induction With $0.5 \%$ Colchicine Solution}

Every 2 h, 2-3 flower buds were randomly collected and fixed in Carnoy's solution (ethanol: acetic acid, 3:1). The anthers were dissected from the fixed buds using forceps and were crushed in a droplet of aceto-carmine solution (2\%) onto a microscope slide to observe meiosis. When the dominant meiotic stage of the PMCs were the leptotene, pachytene, diplotene, diakinesis, and metaphase I stages. The male buds were injected with $0.5 \%$ colchicine solution $3,5,7,9$, and 11 times, respectively. The interval between neighboring injections was $2 \mathrm{~h}$. Each bud was injected with $10 \mu \mathrm{l}$ colchicine solution, and 15-20 flower buds with each treatment were injected. Untreated male buds were taken as a control group. The treated and the control floral branches were hydroponically cultured in a greenhouse for 4 weeks until the catkins matured, then pollen samples were collected and stored in a centrifuge tube with allochronic silica gel at $-20^{\circ} \mathrm{C}$.

The diameter of the $2 \mathrm{n}$ pollen grain is larger than that of haploid pollen grain because of its chromosome doubling in higher plants (De Storme et al., 2012; Zhang and Kang, 2013; Yang et al., 2015). Therefore, we can determine $2 \mathrm{n}$ pollen from haploid pollen by the diameter of pollen grains. Thus, the frequency of $2 n$ pollen was estimated according to the method described by Zhang and Kang (2013). The diameters of $200-300$ pollen grains per sample were measured.

\section{Morphology of Colchicine-Induced 2n Pollen Grains}

For the scanning electron microscopic analysis, pollen samples were sputter-coated with gold for $1 \mathrm{~min}$, using a Hitachi E010 ion sputter coater (Tokyo, Japan). The pollen grains were observed under a Hitachi S-3400N microscope at an accelerating voltage of $5 \mathrm{kV}$. The details of ectexine structure and deposition were observed and photographed for each pollen sample.

\section{Pollen Germination in vitro}

The in vitro pollen germination medium was prepared according to the method described by Zhao et al. (2017). The medium was composed of $0.7 \%$ agar, $50 \mathrm{mg} \mathrm{l}^{-1}$ calcium chloride, and $120 \mathrm{mg} \mathrm{l}^{-1}$ boric acid. The $\mathrm{pH}$ value of the medium was 6.0 . Some fresh colchicine-induced $2 \mathrm{n}$ pollen grains were sampled and spread on slides containing the medium. Then, the slides were placed in $120 \mathrm{~mm}$ Petri dishes with wet filter paper, and the pollen was germinated in a climate chamber at $26^{\circ} \mathrm{C}$. After a $4 \mathrm{~h}$ culture, the pollen grains were washed in a liquid germination medium, collected in a $1.5 \mathrm{ml}$ centrifuge tube, and fixed in Carnoy's solution for $10 \mathrm{~min}$ after being centrifuged for $5 \mathrm{~min}$ at $1,000 \mathrm{rpm}$. The germination rate and the length of each sample 
were calculated using an eyepiece micrometer. A total of 20-30 pollen tubes was assessed in each sample.

\section{Triploid Production by Crossing Colchicine-Induced 2n Pollen}

The female flower buds were pollinated with fresh colchicineinduced $P$. canescens pollen when the stigmas of the female flower buds were receptive. After pollination, the female flower branches were hydroponically cultured. Seeds were collected after 4 weeks and sown into $54 \times 28 \times 10 \mathrm{~cm}$ nutrition plates at a depth of $5 \mathrm{~cm}$ to promote growth, then placed in a greenhouse.

\section{Ploidy Analysis via Flow Cytometry and Somatic Chromosome Counting}

A flow cytometry analysis was conducted by flow cytometer (BD FACSCalibur; Becton Dickinson, Brea, CA, United States). Approximately $0.5 \mathrm{~g}$ of chopped young leaves were put into a $55 \mathrm{~mm}$ Petri dish with $1 \mathrm{ml}$ of nuclear extraction solution $(45 \mathrm{mM}$ $\mathrm{MgCl}_{2}, 30 \mathrm{mM}$ sodium citrate, $20 \mathrm{mM}$ 4-morpholinepropane sulfonate, $1 \%(\mathrm{v} / \mathrm{v})$ Triton $\mathrm{X}-100, \mathrm{pH}=7.0)$, and the nuclei suspension was filtered through a $40 \mu \mathrm{m}$ nylon mesh. The nuclei were stained with $50 \mu \mathrm{l}$ of 4',6-diamidino-2-phenylindole $(10 \mathrm{mg} / \mathrm{ml})$ for $5 \mathrm{~min}$. At least 2,000 nuclei were analyzed, and three samples were collected per plant. Leaves sampled from a known diploid plant of $P$. tomentosa $(2 \mathrm{n}=2 \mathrm{x}=38)$ were taken as a control. The plant DNA C-value of Populus is $0.46 \mathrm{pg}$ (Kew $C$ - value database). The standard peak was set to appear at about channel 50 relative fluorescent intensity. Therefore, when the peaks appeared at channel 75 relative fluorescent intensity, they were considered putative triploids.

We confirmed the ploidy level of each plantlet by somatic chromosome counting. Stem tips were collected and pretreated in a saturated solution of paradichlorobenzene for 2-3 h. Then, the pretreated samples were washed once and fixed in Carnoy's solution at $4^{\circ} \mathrm{C}$ for at least $24 \mathrm{~h}$. Subsequently, the fixed stem tips were hydrolyzed in $38 \% \mathrm{HCl}$ for $25 \mathrm{~min}$ at room temperature and then rinsed three times for $10 \mathrm{~min}$ each with distilled water. The hydrolyzed samples were stained with Carbol fuchsin, crushed with a coverslip, and observed at $100 \times$ oil lens magnification in an Olympus BX51 microscope (Olympus Inc., Tokyo, Japan).

\section{Identification of Genetic Constitutions of Colchicine-Induced 2n Pollen}

According to the manufacturer's protocol, DNA was extracted from a $300 \mathrm{mg}$ stored leaf sample of each plant, including the parental poplar lines and their triploid hybrids using the DNeasy Plant Mini Kit (Tiangen Biotech) ${ }^{1}$. The fluorescently labeled TP-M13-SSR method (Schuelke, 2000) was employed in the present study. A forward primer at the $5^{\prime}$ end was attached with a universal primer M13 tail (5'-TGTAAAACGACGGCCAGT- $\left.3^{\prime}\right)$ and labeled with four fluorescent substances (6-carboxy-X-rhodamine, 6carboxy-fluorescein, tetramethyl-6-carboxyrhodamine, or 5-hexachloro-fluorescein). All primers were synthesized by

${ }^{1}$ http://www.tiangen.com/en/?app.html
Sangon Inc. ${ }^{2}$ The PCR amplification protocol was as follows: $5 \mathrm{~min}$ at $94^{\circ} \mathrm{C} ; 10$ cycles of $30 \mathrm{~s}$ at $94^{\circ} \mathrm{C}, 30 \mathrm{~s}$ at the optimal annealing temperature for each SSR marker, and $30 \mathrm{~s}$ at $72^{\circ} \mathrm{C} ; 25$ cycles of $30 \mathrm{~s}$ at $94^{\circ} \mathrm{C}, 30 \mathrm{~s}$ at $53^{\circ} \mathrm{C}$, and $30 \mathrm{~s}$ at $72^{\circ} \mathrm{C}$; and a final extension of $8 \mathrm{~min}$ at $72^{\circ} \mathrm{C}$. Then the PCR products were used for capillary electrophoresis fluorescence-based SSR analyses using the ABI 3730xl DNA Analyzer (Applied Biosystems, Foster City, CA, United States), and fragment sizes and peak areas analyzed using GeneMarker software V1.75 (Hulce et al., 2011).

We screened SSR primers from the SSR database ${ }^{3}$ (beginning with "GCPM," "ORPM", and "PMGC") released by the International Populus Genome Consortium (IPGC). BLAST analysis in Populus trichocarpa v3.0 (Phytozome v12.0) ${ }^{4}$ was done to determinate the location of the SSR loci.

\section{Statistical Analysis}

We analyzed the frequency of colchicine-induced $2 n$ pollen using a univariate general linear model (GLM) to reveal the differences among the meiotic stages, injection times, and the interaction between the meiotic stage and injection time. The data were transformed before analysis of variance and multiple comparisons to account for the heterogeneity of variance. All statistical analyses were performed with SPSS software (version 18.0; SPSS Inc., Chicago, IL, United States).

\section{RESULTS}

\section{2n Pollen Production Using 0.5\% Colchicine Solution}

After the sampled male buds were injected with $0.5 \%$ colchicine solution at leptotene (Figure 1A), pachytene (Figure 1B), diplotene (Figure 1C), diakinesis (Figure 1D), or metaphase I (Figure 1E), the treated male $P$. canescens flower buds were hydroponically cultured in a greenhouse until the catkins matured. As the pollen was released, colchicine-induced $2 \mathrm{n}$ pollen was collected from the treated male buds (Figure 1F). A few naturally occurring $2 \mathrm{n}$ pollen grains were found in the control group (Figure 1G). The frequency of colchicine-induced 2 n pollen ranged from 2.75 to $30.27 \%$ (Table 1). We found that the dominant meiotic stage $(F=4.801, P=0.009)$, injection time $(F=12.449, P=0.000)$, and the interaction between the dominant meiotic stage and injection time $(F=3.006, P=0.002)$ had highly significant effects on the frequency of colchicine-induced 2n pollen according to the univariate GLM analysis. Further LSD multiple-comparison tests indicated that the differences in colchicine-induced $2 \mathrm{n}$ pollen frequency were significantly higher at pachytene than those at the leptotene, diplotene, or metaphase I stages $(p<0.05)$. The differences in the frequency of colchicine-induced $2 \mathrm{n}$ pollen for samples given 11 injections was also significantly higher than that of those given 3, 5, 7, and 9 injections. Hence, the optimal protocol for inducing $2 \mathrm{n}$ pollen via

\footnotetext{
${ }^{2}$ http://www.life-biotech.com/sangonEn.html

${ }^{3} \mathrm{http}: / /$ popgenie.org

${ }^{4}$ https://phytozome.jgi.doe.gov/pz/portal.html
} 


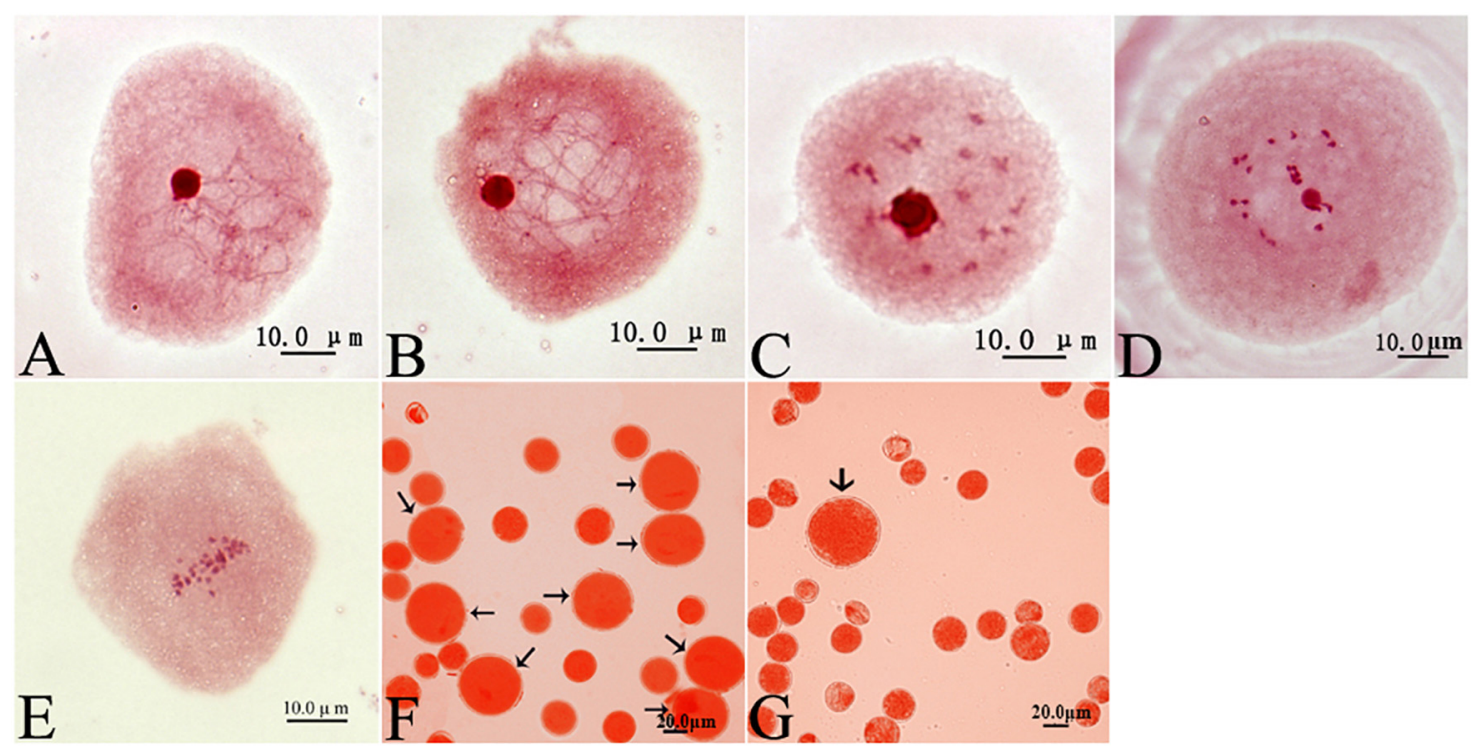

FIGURE 1 | Cytological observations of PMCs, colchicine-induced 2n pollen (arrow) and naturally occurring 2n pollen in Populus canescens. (A) Leptotene. (B) Pachytene. (C) Diplotene. (D) Diakinesis. (E) Metaphase I. (F) Colchicine-induced 2n pollen derived from the treatment given 11 injections at pachytene in P. canescens (arrow). (G) Natural 2n pollen derived from the control group in P. canescens (arrow). Scale bar $=10.0 \mu \mathrm{m}(\mathbf{A}-\mathbf{E})$ and $20.0 \mu \mathrm{m}$ (F,G).

colchicine was to give 11 injections of $0.5 \%$ colchicine solution at the pachytene stage of the PMCs.

\section{The Effects of Colchicine Treatment on Pollen Morphology}

The morphology of the pollen was examined via scanning electron microscopy (Figure 2). The pollen grains were nonspherical, with few corrugations and granulated decorations on the surface. $2 \mathrm{n}$ pollen was observed in the control group (Figure 2A), the 5 injections group (Figure 2B), and the 11 injections group at pachytene (Figure 2C). The haploid pollen was homogenous, with granulated decorations on the surface, and no aperture was observed in the control or treatment groups (Figures 2D-I). No significant differences were observed in the ectexine structure of haploid pollen between the control and treatment groups because haploid pollen grains were not the outcome of colchicine treatment. However, compared with natural $2 \mathrm{n}$ pollen in the control group (Figure 2J), the morphology of the colchicine-induced 2 n pollen (Figures $2 \mathrm{~K}, \mathrm{~L}$ ) showed altered ectexine deposition and some narrow furrows in the ectexine structure.

\section{Triploid Production by Crossing Colchicine-Induced 2n Pollen}

A total of 6,741 mature seeds were collected from 2 crossbreeding combinations of colchicine-induced $2 \mathrm{n}$ pollen and the control group. All seeds were sown and became 4955 young seedlings. Among them, 76 seedlings were derived from the cross between $P$. hopeiensis and $P$. canescens, and 4,879 seedlings were derived from the cross between $P$. alba $\times P$. glandulosa and $P$. canescens. The ploidy level of all offspring was examined by flow cytometry, and five putative triploids were detected (Figure 3A). Subsequently, the five putative triploids were confirmed by somatic chromosome counts. The number of chromosomes in the diploid was $38(2 \mathrm{n}=2 \mathrm{x}=38$, Figure $3 \mathrm{~B})$, and the number of chromosomes in the triploid was 57 $(2 \mathrm{n}=3 \mathrm{x}=57$, Figure $3 \mathrm{C})$, indicating that the five putative triploids were real triploids. Among the five triploids, two came from $P$. hopeiensis $\times P$. canescens, and three were derived from $(P$. alba $\times P$. glandulosa $) \times P$. canescens.

The number of hybrid offspring and the triploid production rates in the different crosses are shown in Table 2. All triploids were derived from crossing the colchicine-induced $2 \mathrm{n}$ pollen with haploid eggs of $P$. hopeiensis or $P$. alba $\times P$. glandulosa. No triploid was detected in most of the cross combinations. The highest triploid production rate (16.67\%) was observed in the cross between $P$. hopeiensis and $P$. canescens. The average production rate of triploids was $2.35 \%$, which was significantly lower than the frequency of colchicine-induced $2 \mathrm{n}$ pollen. These results suggest that the competitive ability of colchicine-induced $2 \mathrm{n}$ pollen was significantly weaker than that of haploid pollen.

\section{Pollen Germination in vitro}

The induced pollen of each treatment contained $2 \mathrm{n}$ and haploid pollen because the meiosis of PMCs in P. canescens is an asynchronous process (Tian M. et al., 2018). We conducted pollen germination experiments in vitro to evaluate the effect of colchicine on induced $2 \mathrm{n}$ pollen viability. The fresh colchicineinduced pollen grains derived from the 11-injections treatment and the fresh pollen from the control group were used for the germination test on medium containing $50 \mathrm{mg} / \mathrm{L}$ calcium chloride and $120 \mathrm{mg} / \mathrm{L}$ boric acid. After a $4 \mathrm{~h}$ culture, some of the colchicine-induced pollen grains had germinated (Figure 4A). 
TABLE 1 | Induction of 2 n pollen via a $0.5 \%$ colchicine solution in $P$. canescens.

\begin{tabular}{lcc}
\hline $\begin{array}{l}\text { Dominant } \\
\text { meiotic stage } \\
\text { of PMCs }\end{array}$ & $\begin{array}{c}\text { No. of colchicine } \\
\text { injections times }\end{array}$ & $\begin{array}{c}\text { Frequency of } \\
\text { colchicine-induced 2n } \\
\text { pollen (\%) }\end{array}$ \\
\hline Leptotene & 3 & $4.79 \pm 1.46$ \\
& 5 & $6.42 \pm 2.13$ \\
& 7 & $9.40 \pm 1.32$ \\
Pachytene & 9 & $8.19 \pm 0.99$ \\
& 11 & $10.44 \pm 4.41$ \\
& 3 & $5.46 \pm 0.90$ \\
Diplotene & 5 & $6.22 \pm 0.89$ \\
& 7 & $9.04 \pm 2.62$ \\
& 9 & $16.70 \pm 3.95$ \\
& 11 & $30.27 \pm 8.69$ \\
& 3 & $4.18 \pm 1.52$ \\
Diakinesis & 5 & $5.79 \pm 0.60$ \\
& 7 & $8.76 \pm 0.45$ \\
& 9 & $9.48 \pm 1.31$ \\
Metaphase I & $9.68 \pm 1.76$ \\
& 11 & $6.99 \pm 1.31$ \\
& 3 & $7.81 \pm 0.74$ \\
& 5 & $8.17 \pm 0.56$ \\
& 7 & $10.53 \pm 0.82$ \\
9 & $20.36 \pm 1.49$ \\
& 11 & $2.75 \pm 0.43$ \\
& 3 & $3.91 \pm 1.53$ \\
& 5 & $11.03 \pm 1.23$ \\
& & $2.08 \pm 0.40$ \\
& &
\end{tabular}

The average pollen germination rate was $27.1 \%$. Some germinated colchicine-induced $2 \mathrm{n}$ pollen grains (Figure $4 \mathrm{~B}$ ) and a few germinated natural $2 \mathrm{n}$ pollen grains (Figure $\mathbf{4 C}$ ) were found. The average germination rates of natural $2 \mathrm{n}$ pollen and induced $2 \mathrm{n}$ pollen were 23.9 and $22.6 \%$, respectively. However, no significant differences were observed in the germination rates between induced $2 \mathrm{n}$ pollen and natural $2 \mathrm{n}$ pollen (Figure 5). The length of the pollen tube in the induced $2 \mathrm{n}$ pollen was $61.9 \pm 9.0 \mu \mathrm{m}$, which was significantly shorter than that of haploid pollen $(279.3 \pm 10.9 \mu \mathrm{m})$ (Figure 6), suggesting that the slow-growing pollen tube of the colchicine-induced $2 \mathrm{n}$ pollen is responsible for the low triploid production rate in $P$. canescens.

\section{Identification the Genetic Composition of Colchicine-Induced 2n Pollen}

To identify the genetic composition of colchicine-induced $2 \mathrm{n}$ pollen, two pairs of polymorphic SSR primers, GCPM_1158 and GCPM_124, were screened. Table 3 presents the detailed information of the two pairs of SSR primers. The GCPM_124 and the GCPM_1158 loci are located on chromosomes 1 (Chr 01) and 2 (Chr 02), respectively. The capillary electrophoresis results showed that the allelic configuration was, at the GCPM_1158 locus, homozygous in the female parent "HBY1" (225.0 bp, for "aa"), and heterozygous in the male parent "YHY1" (240.8 bp and $253.0 \mathrm{bp}$, for "b" and "c") (Figure 7A). The resulting genetic constitution of T1 and T2 triploid hybrids was proven to be "abc." Therefore, the triploid progeny T1 and T2 could be from FDR-type 2n pollen. Similar to the GCPM_1158 locus, the allelic configuration was, at the GCPM_124 locus, homozygous in female parent "YXY1" (212.3 bp for "aa"), and heterozygous in the male parent "YHY1" (200.6 bp and $206.3 \mathrm{bp}$, for "b" and "c") (Figure 7B). The genetic constitution of triploid hybrids T3, T4, and T5, was found to be "abc." Consequently, triploid progeny T3, T4, and T5 could be inferred to derive from FDRtype $2 \mathrm{n}$ pollen.

\section{DISCUSSION}

Colchicine is considered an effective chemical mutagen to induce diploid gametes in plants. It inhibits microtubule polymerization by binding to tubulin, and thus it inhibits the mitotic spindle formation resulting in the development of a polyploid cell. Colchicine has been successfully used to induce $2 \mathrm{n}$ female gametes by treating embryo sacs, zygotes, and somatic cells, furthermore, a large number of triploids and tetraploids have been generated in Populus (Li et al., 2001; Kang et al., 2004; Wang et al., 2010a,b; Cai and Kang, 2011; Xu et al., 2016), Robinia pseudoacacia (Ewald et al., 2009), Eucommia ulmoides (Zhang et al., 2008), and Eucalyptus urophylla (Yang et al., 2018). Colchicine has also been applied to induce $2 \mathrm{n}$ pollen production in Populus (Li et al., 2014; Zhao et al., 2017, 2019), Eucommia ulmoides (Gao et al., 2004), and Eucalyptus (Yang et al., 2016). Several triploids have been created by crossing colchicineinduced $2 \mathrm{n}$ pollen in Populus (Zhao et al., 2017). Therefore, triploid breeding is a promising way to improve Populus.

Applying colchicine to PMCs at a suitable stage is vital for inducing $2 \mathrm{n}$ pollen (Kang, 2016). The most suitable stage is species dependent. Kang et al. (1999) reported that the most suitable stage for inducing $2 \mathrm{n}$ pollen via colchicine varies from leptotene to diplotene in $P$. tomentosa $\times P$. bolleana. The leptotene to pachytene stage is the most effective for inducing $2 \mathrm{n}$ pollen by colchicine in P. alba (Li et al., 2014). In our study, the most effective stage for inducing $2 \mathrm{n}$ pollen was pachytene during microsporogenesis of $P$. canescens, which was consistent with the findings reported by Zhao et al. (2019) for P. deltoid.

The number of colchicine injections is key when inducing 2n pollen in Populus. According to previous reports, the suitable number of colchicine injections varies from 3 to 5 in $P$. tomentosa $\times P$. bolleana for the highest frequency of induced 2n pollen (88\%) (Kang et al., 1999). Zhao et al. (2017) documented that $56.5 \%$ of induced $2 \mathrm{n}$ pollen is achieved when the male $P$. tomentosa buds are given 7 injections of colchicine. In the present study, 11 colchicine injections to the male buds of $P$. canescens at pachytene were used. The highest frequency of induced $2 \mathrm{n}$ pollen was $30.27 \%$, which was significantly lower than that of previous studies (Kang et al., 1999; Zhao et al., 2017). At least two aspects are responsible for the lower frequency of induced $2 \mathrm{n}$ pollen. The meiotic process of the PMCs in different anthers inside the same flower bud is asynchronous (Tian M. et al., 2018). When the meiotic stage of PMCs in P. canescens was 


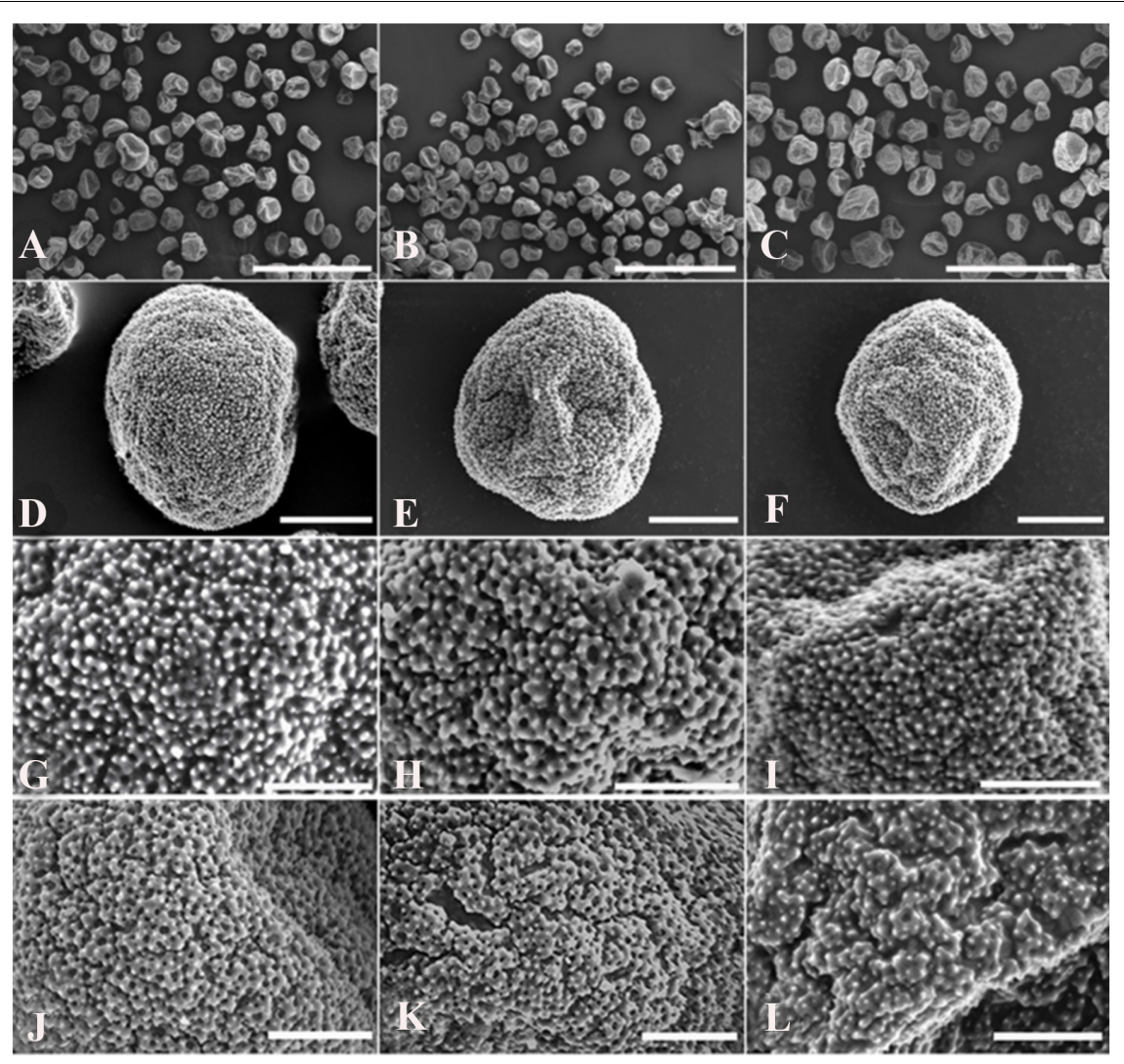

FIGURE 2 | Scanning electron micrographs of $P$. canescens pollen grains derived from untreated and treated male flower buds with $0.5 \%$ colchicine at pachytene. (A) Morphology of the pollen grains in the control group and natural 2n pollen grains. (B) Morphology of the pollen grains derived from the treatment given 5 injections at pachytene and induced $2 \mathrm{n}$ pollen grains. (C) Morphology of the pollen grains derived from the treatment given 11 injections at pachytene and induced 2n pollen grains. (D-F) Ectexine deposition; (D) haploid pollen grains in the control group; (E) haploid pollen grains derived from the 5 injections treatment at pachytene; (F) haploid pollen grains derived from the 11 injections treatment given at pachytene. (G-L) Details of the ectexine structure; (G) haploid pollen grains in the control group; $\mathbf{( H )}$ haploid pollen grains derived from the treatment given 5 injections at pachytene; (I) haploid pollen grains derived from the treatment given 11 injections at pachytene; (J) natural 2n pollen grains; (K) 2n pollen grains derived from the treatment given 5 injections at pachytene; (L) $2 n$ pollen grains derived from the treatment given 11 injections at pachytene. Scale bar $=100 \mu \mathrm{m}$ (A-C), $10 \mu \mathrm{m}$ (D-F), $5 \mu \mathrm{m}$ (G-L).
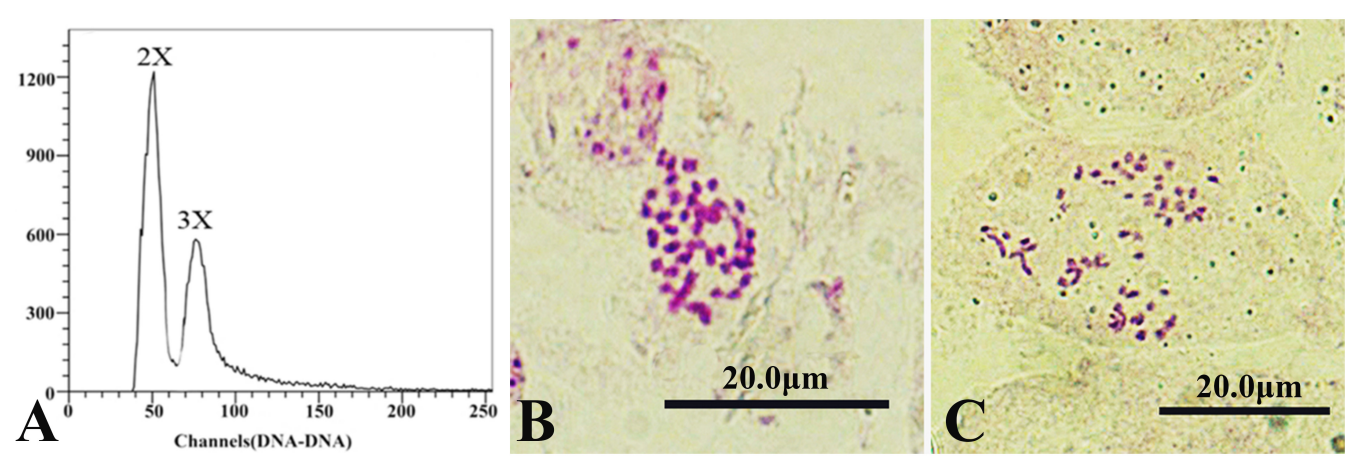

FIGURE 3 | Flow cytometric analysis and somatic chromosome counting of the offspring derived from pollen chromosome doubling induced by colchicine in P. canescens (scale bar $=20 \mu \mathrm{m}$ ). (A) Flow cytometric analysis of the mixed simple of a diploid plant and a triploid plant; (B) Chromosome number of the diploid $(2 n=2 x=38)$; (C) Chromosome number of the triploid $(2 n=3 x=57)$.

at pachytene, the catkin had emerged from the bract scales. The effect of colchicine treatment decreased because the colchicine overflowed and evaporated (Figure 8).
Heterozygosity within a $2 \mathrm{n}$ pollen grain depends on the cytological mechanism governing $2 \mathrm{n}$ pollen formation. These mechanisms are subdivided into first division restitution (FDR), 
TABLE 2 | Triploid production by crossing colchicine-induced $P$. canescens $2 \mathrm{n}$ pollen with haploid female gametes in $P$. hopeiensis and $P$. alba $\times$ P. glandulosa.

\begin{tabular}{|c|c|c|c|c|c|}
\hline Female parents & $\begin{array}{c}\text { Frequency of } \\
\text { induced } 2 n \text { pollen }(\%)\end{array}$ & $\begin{array}{l}\text { Number of } \\
\text { seeds }\end{array}$ & $\begin{array}{l}\text { Number of } \\
\text { seedlings }\end{array}$ & $\begin{array}{l}\text { Number of } \\
\text { triploids }\end{array}$ & $\begin{array}{l}\text { Triploid production rates } \\
\text { (\%) }\end{array}$ \\
\hline \multirow[t]{6}{*}{ P. hopeiensis } & 30.27 & 38 & 6 & 1 & 16.67 \\
\hline & 20.36 & 33 & 9 & 1 & 11.11 \\
\hline & 16.70 & 64 & 21 & 0 & 0 \\
\hline & 15.11 & 31 & 16 & 0 & 0 \\
\hline & 11.03 & 52 & 20 & 0 & 0 \\
\hline & 10.53 & 27 & 4 & 0 & 0 \\
\hline \multirow[t]{8}{*}{ P. alba $\times P$. glandulosa } & 9.05 & 912 & 610 & 2 & 0.33 \\
\hline & 8.76 & 1027 & 864 & 1 & 0.12 \\
\hline & 6.22 & 704 & 563 & 0 & 0 \\
\hline & 5.79 & 975 & 623 & 0 & 0 \\
\hline & 5.46 & 1217 & 964 & 0 & 0 \\
\hline & 4.34 & 694 & 552 & 0 & 0 \\
\hline & 2.08 (control) & 967 & 703 & 0 & 0 \\
\hline & total & 6741 & 4955 & 5 & \\
\hline
\end{tabular}

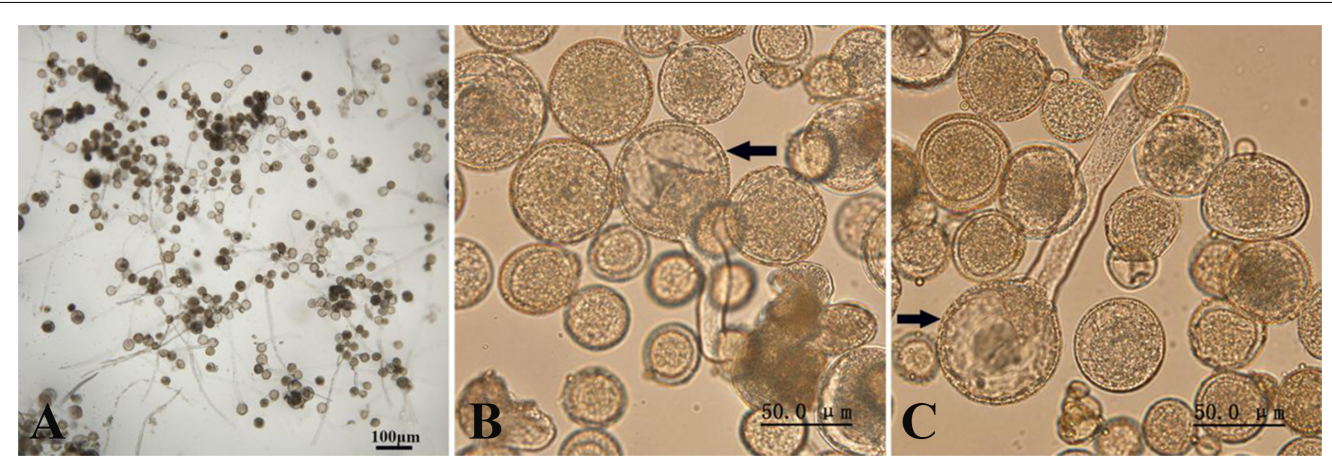

FIGURE 4 | Fresh colchicine-induced pollen of $P$. canescens derived from the treatment given 11 injection times germinated on media. (A) Germinated fresh colchicine-induced pollen derived from the treatment given 11 injection times. (B) Germinated $2 n$ pollen induced by colchicine (arrow). (C) Germinated natural $2 n$ pollen (arrow). Scale bar = 100.0 (A) and $50.0 \mu \mathrm{m}$ (B,C).

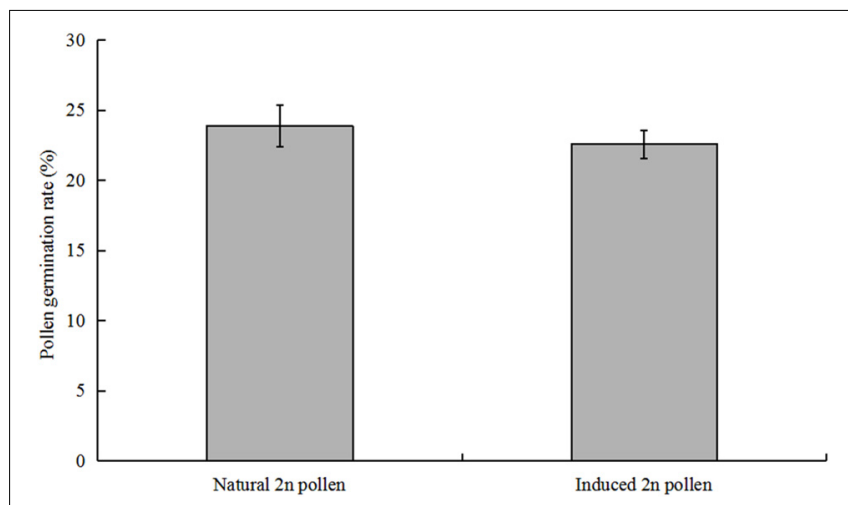

FIGURE 5 | Germination rates of natural 2n pollen derived from the control group and colchicine-induced $2 \mathrm{n}$ pollen derived from the treatment given 11 injections with $0.5 \%$ colchicine at pachytene in $P$. canescens. second division restitution (SDR) (Ramanna and Jacobsen, 2003), and indeterminate meiotic restitution (IMR) (Lim et al., 2001). Pairing and separation of the homologous chromosomes

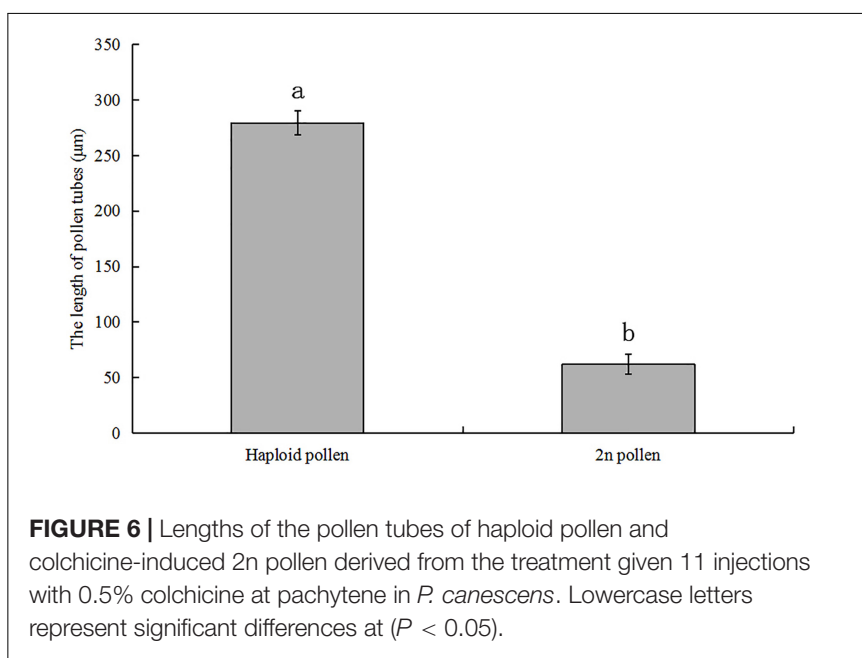

does not occur at meiosis I during FDR, whereas the second division occurs normally with the two sister chromatids of each chromosome moving to opposite poles. Thus, except for 
TABLE 3 | The detailed information of the two pairs of polymorphic SSR primers and annealing temperature (AT).

\begin{tabular}{llll}
\hline ID of SSR primer & Chromosome & \multicolumn{2}{c}{ SSR primer sequence } \\
\cline { 3 - 4 } & & Forward & Reverse \\
\hline GCPM_124 & Chr 01 & TTGAGCACTTCAACTACCA & TGTCTTCCCTTAGTCACCAC \\
GCPM_1158 & Chr 02 & ATGCACTTCCTTCCAAATA & ATCAGTTCCTTCAGCTTCAA
\end{tabular}

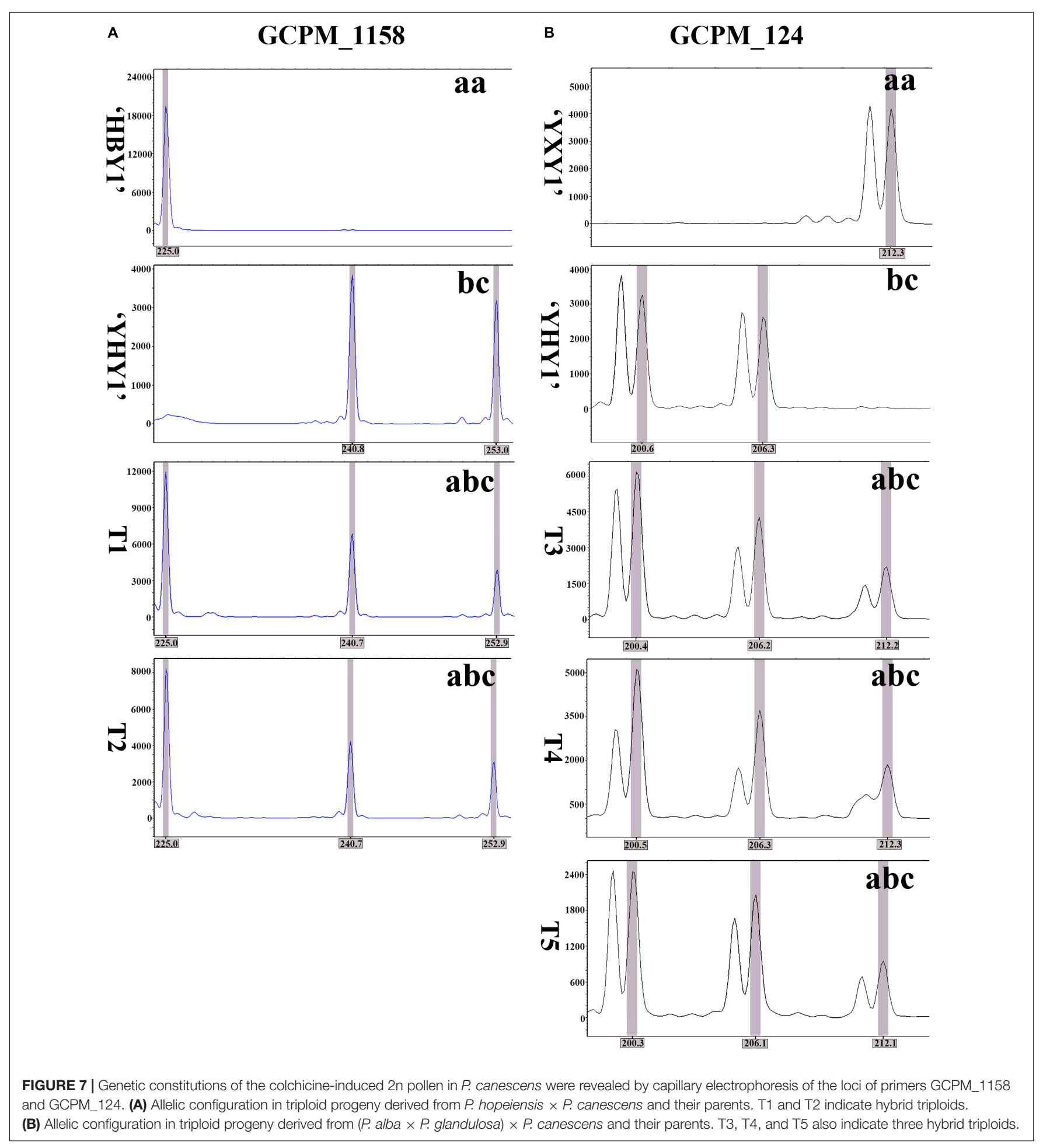




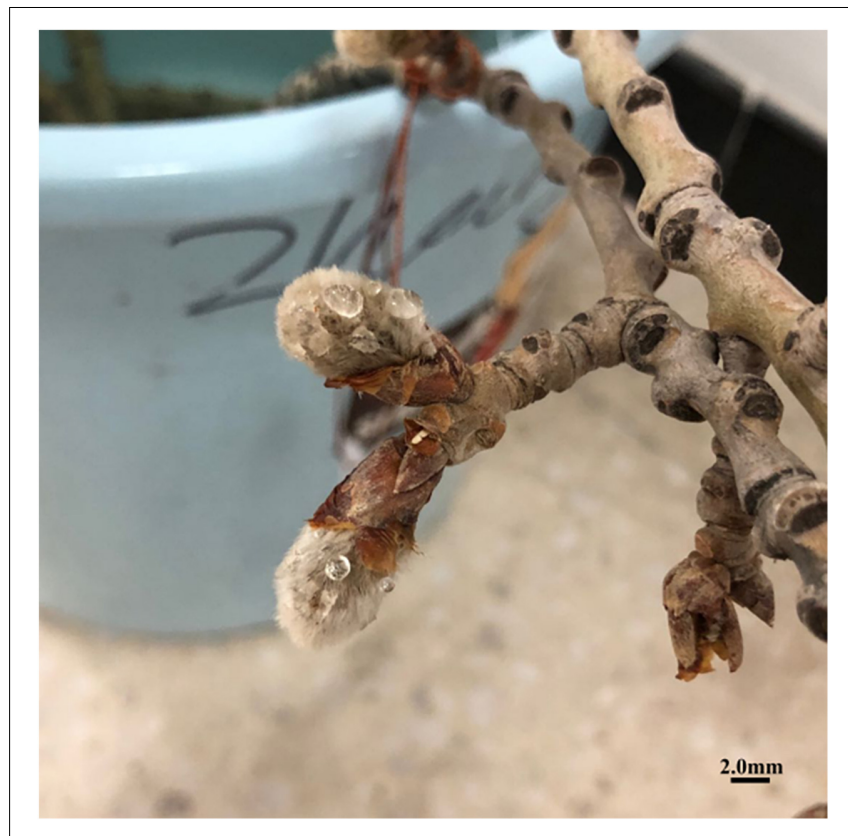

FIGURE 8 | Male flower buds of $P$. canescens after treatment with $0.5 \%$ colchicine solution. Scale bar $=2.0 \mathrm{~mm}$.

cross-over segments, FDR-type $2 \mathrm{n}$ pollen retain all homologous parental chromosomes. FDR-type $2 \mathrm{n}$ pollen is important when creating heterozygous hybrids due to the highly heterozygous 2n gametes formed (Bretagnolle and Thompson, 1995). In SDR, the pairing and separation of the homologous chromosomes proceed normally during meiosis. In meiosis II, the centromeres of the half-bivalents divide, but the chromatids do not migrate to the poles. Therefore, heterozygosity within SDR-type $2 \mathrm{n}$ pollen is lower than that within FDR-type $2 \mathrm{n}$ pollen because SDRtype $2 \mathrm{n}$ gametes only contain random combinations of sister chromatids. Another restitution mechanism of $2 \mathrm{n}$ pollen is IMR. In IMR, unequal distribution of the centromeres of the parental genomes occurs at metaphase I, where some univalents are divided equationally during the first meiotic process, whereas the remaining bivalents are separated before the end of meiosis giving rise to 2n-gametes that cannot be categorized as either FDR or SDR. The IMR mechanism is the process of meiotic restitution to produce fully heterozygous $2 \mathrm{n}$ gametes.

Several studies have reported the mechanisms of the formation of $2 \mathrm{n}$ gametes in Populus. Both FDR-type and SDR-type $2 \mathrm{n}$ megaspores are obtained by treating female flower buds with high temperature during megasporogenesis in P. pseudo-simonii $\times$ P. nigra "Zheyin \#3" (Wang et al., 2012), P. adenopoda (Lu et al., 2013), and P. tomentosa (Min et al., 2017). Dong et al. (2015) demonstrated that $74.8 \%$ of parental heterozygosity was transmitted by FDR-type $2 \mathrm{n}$ female gametes, and $39.6 \%$ of parental heterozygosity was transmitted by SDR-type $2 \mathrm{n}$ female gametes in $P$. pseudosimonii $\times P$. nigra "Zheyin \#3." However, $\operatorname{Lim}$ et al. (2001) and Barba-Gonzalez et al. (2005) reported that IMR-type $2 \mathrm{n}$ gametes were detected in interspecific lily hybrids by GISH and FISH. SDR- and PMD -type (postmeiotic genome doubling) $2 \mathrm{n}$ gametes were also detected by cytological observation and SSR makers in "Eureka" lemon (Xie et al., 2019). In the present study, the allelic configuration, at the GCPM_1158 and GCPM_124 loci, revealed that the five triploids originated from FDR-type induced $2 \mathrm{n}$ pollen. Therefore, the colchicine-induced $2 \mathrm{n}$ pollen grains were FDR-type when colchicine treatment occurred before metaphase I.

High-temperature is one of most effective physical mutagenic agents and is widely used to induce 2 n gametes in higher plants (Pécrix et al., 2011; Tian M. et al., 2018). Many studies have reported changes in pollen wall structure (Porch and Jahn, 2001; Koti et al., 2005) and a decrease in pollen viability (Porch and Jahn, 2001). For example, $36^{\circ} \mathrm{C}$ exposure during early meiosis of PMCs leads to a decrease in pollen viability and pollen ectexine defects in induced $2 \mathrm{n}$ pollen of Rosa (Pécrix et al., 2011). Tian M. et al. (2018) showed a decrease in the pollen production per male flower bud when $P$. canescens buds are exposed to $38^{\circ} \mathrm{C}$ for $6 \mathrm{~h}$, and the morphology of the induced $2 \mathrm{n}$ pollen revealed narrow furrows in the ectexine structure. However, the germination rate of induced $2 \mathrm{n}$ pollen was not significantly affected.

It is unknown whether colchicine treatment affects the pollen wall structure and induced $2 \mathrm{n}$ pollen viability in Populus. Liu et al. (2019) reported that the ectexine structure of $P$. tomentosa induced $2 \mathrm{n}$ pollen by giving 3 or 7 colchicine injections at diakinesis of PMCs was similar to that of the natural $2 \mathrm{n}$ pollen. In the present study, the morphology of induced $2 \mathrm{n}$ pollen was observed by scanning electron microscopy to clarify whether colchicine affected induced $2 n$ pollen viability in $P$. canescens. Some narrow furrows were found in the ectexine structure of colchicine-induced $2 n$ pollen. However, no significant difference in germination rates was detected between colchicine-induced $2 \mathrm{n}$ pollen and natural $2 \mathrm{n}$ pollen, and five triploids were detected by flow cytometry and somatic chromosome counts, suggesting that colchicine treatment will not result in dysfunction of induced $2 \mathrm{n}$ pollen.

In this study, the triploid production rates were significantly lower than the frequency of colchicine-induced $2 \mathrm{n}$ pollen, indicating that $2 \mathrm{n}$ pollen weakly competed with haploid pollen during fertilization. The in vitro pollen germination experiments revealed that the reason the induced $2 n$ pollen was weakly competitive during fertilization was because the pollen tubes of colchicine-induced $2 \mathrm{n}$ pollen grew slower than those of haploid pollen. Our findings are consistent with Zhao et al. (2017) in male $P$. tomentosa clone 5088. In a future study, we will focus on revealing the precise reason pollen-tubes of $2 \mathrm{n}$ pollen grow so slowly in P. canescens.

\section{CONCLUSION}

The meiotic stage, injection time, and the interaction between the meiotic stage and injection time had highly significant effects on $2 \mathrm{n}$ pollen production rates in $P$. canescens. 
The most effective treatment for inducing $2 \mathrm{n}$ pollen was to give 11 injections of $0.5 \%$ colchicine solution when PMCs were at the pachytene stage. Colchicine occasionally affected ectexine deposition, and some narrow furrows were detected in the ectexine structure. However, no significant difference in pollen germination rates was observed between natural $2 \mathrm{n}$ pollen and colchicine-induced $2 \mathrm{n}$ pollen, and five triploids derived from FDR-type induced $2 n$ pollen were generated by crossing colchicine-induced $2 \mathrm{n}$ pollen, suggesting that colchicine will not result in dysfunction of colchicine-induced $2 \mathrm{n}$ pollen. Slower growth of $2 \mathrm{n}$ pollen tubes was responsible for the lower triploid production rate.

\section{DATA AVAILABILITY STATEMENT}

All datasets generated for this study are included in the article/supplementary material.

\section{REFERENCES}

Barba-Gonzalez, R., Ramanna, M. S., Visser, R. G., Visser, R. G. F., and Tuyl, J. M. V. (2005). Intergenomic recombination in F1 lily hybrids (Lilium) and its significance for genetic variation in the $\mathrm{BC} 1$ progenies as revealed by GISH and FISH. Genome 48, 884-894. doi: 10.1139/g05-057

Bretagnolle, F., and Thompson, J. D. (1995). Gametes with the somatic chromosome number: mechanisms of their formation and role in the evolution of autoploid plants. New Phytol. 129, 1-22. doi: 10.1111/j.1469-8137.1995. tb03005.x

Cai, X., and Kang, X. Y. (2011). In vitro tetraploid induction from leaf explants of Populus pseudo-simonii Kitag. Plant Cell Rep. 30, 1771-1778. doi: 10.1007/ s00299-011-1085-z

De Storme, N., Copenhaver, G. P., and Geelen, D. (2012). Production of diploid male gametes in arabidopsis by cold-induced destabilization of postmeiotic radial microtubule arrays. Plant Physiol 160, 1808-1826. doi: 10.1104/pp.112. 208611

Dong, C. B., Suo, Y. J., Wang, J., and Kang, X. Y. (2015). Analysis of transmission of heterozygosity by $2 \mathrm{n}$ gametes in Populus (Salicaceae). Tree Genet. Genomes 11:799. doi: 10.1007/s11295-014-0799-9

Duan, A. A., and Yang, M. S. (1998). Physiological determination on cold and drought resistance of $($ Populus tomentosa $\times$ P. bolleana $) \times(P$. alba $\times$ P.tremula $)$ double hybrid clones. Yunnan For Sci. Techn. 84, 27-31. doi: 10.16473/j.cnki. xblykx1972.1998.03.004

Ewald, D., Ulrich, K., Naujoks, G., and Schröder, M. B. (2009). Induction of tetraploid poplar and black locust plants using colchicine: chloroplast number as an early marker for selecting polyploids in vitro. Plant Cell Tiss Org. 99, 353-357. doi: 10.1007/s11240-009-9601-3

Gao, P., Lin, W., and Kang, X. Y. (2004). Pollen chromosome doubling of Eucommia ulmoides induced by colchicine. J. Beijing For. Univ. 26, 39-42. doi: 10.13332/j.1000-1522.2004.04.008

Hulce, D., Li, X., and Snyderleiby, T. (2011). Gene-Marker ${ }^{\circledR}$ genotyping software: tools to increase the statistical power of DNA fragment analysis. J. Biomol. Tech. 22(Suppl.), S35.

Kang, X. Y. (2016). Some understandings on polypliod breeding of poplars. J. Beijing For. Univ. 32, 149-153. doi: 10.13332/j.1000-1522.2010.05.028

Kang, X. Y., Zhang, P. D., Gao, P., and Zhao, F. (2004). Discovery of a new of poplar triploids induced with colchicine after pollination. J. Beijing For. Univ. 26, 1-4. doi: 10.13332/j.1000-1522.2004.01.001

Kang, X. Y., and Zhu, Z. T., and Lin, H. B. (1999). Study on effective treating period for pollen chromosome doubling of Populus tomentosa $\times$ P. bolleana. Sci. Silva Sin. 35, 21-24. doi: 10.3321/j.issn:1001-7488.1999.04.004

\section{AUTHOR CONTRIBUTIONS}

$\mathrm{PZ}$ designed the experiments and edited the language of the manuscript. QZ, JW, YS, and ZZ performed the experiments. QZ performed the data analysis and wrote the manuscript. ML contributed the tools for analysis. All authors read and approved the final manuscript.

\section{FUNDING}

This study was mainly supported by the National Key Research and Development Program of China (2016YFD0600403).

\section{ACKNOWLEDGMENTS}

We thank technician Junna Shi for her assistance in scanning electron microscopic analysis.

Koti, S., Reddy, K. R., Reddy, V. R., Kakani, V. G., and Zhao, D. L. (2005). Interactive effects of carbon dioxide, temperature, and ultraviolet- $\mathrm{B}$ radiation on soybean (Glycine max L.) flower and pollen morphology, pollen production, germination, and tube lengths. J. Exp. Bot. 56, 725-736. doi: 10.1093/jxb/eri044

Li, Y., Guo, Q., Wang, J., Tian, J., and Kang, X. Y. (2014). Colchicine-induced pollen chromosome doubling and its cytological effects in Populus alba L. J. Nucl. Agric. Sci. 28, 749-756. doi: 10.11869/j.issn.100-8551.2014.05.0749

Li, Y., Zhu, Z. T., Tian, Y. T., Zhang, Z. Y., and Kang, X. Y. (2001). Studies on obtaining triploids by colchicine treating female flower buds of white poplar. Sci. Silva Sin. 37, 68-74. doi: 10.3321/j.issn:1001-7488.2001.05.012

Li, T. Q., and Zhu, Z. T. (1989). Study on difficulty of hybridization and patterns of crossing within Leuce. J. Beijing For. Univ. 11, 54-59. doi: 10.13332/j.10001522.1989.03.009

Lim, K. B., Ramanna, M. S., Jong, J. H. D., Jacobsen, E., and Tuyl, J. M. V. (2001). Indeterminate meiotic restitution (IMR): a novel type of meiotic nuclear restitution mechanism detected in interspecific lily hybrids by GISH. Theor. Appl. Genet. 103, 219-230. doi: 10.1007/s001220100638

Liu, Y., Zhang, Y., Zhou, Q., Wu, J., and Zhang, P. D. (2019). Colchicine did not affect the viability of induced 2 n pollen in Populus tomentosa. Sliva Fenn 53, 1-13. doi: 10.14214/sf.10132

Lu, M., Zhang, P. D., and Kang, X. Y. (2013). Induction of 2n female gametes in Populus adenopoda maxim by high temperature exposure during female gametophyte development. Breeding Sci. 63, 96-103. doi: 10.1270/jsbbs.63.96

Min, Y., Kang, N., Suo, Y. J., Tian, J., and Kang, X. Y. (2017). Origin identification of $2 \mathrm{n}$ female gamete of Populus tomentosa triploid hybrids. J. Beijing For. Univ. 39, 17-24. doi: 10.13332/j.1000-1522.20170001

Müntzing, A. (1936). The chromosomes of a giant Populus tremula. Hereditas 21, 383-393. doi: 10.1111/j.1601-5223.1936.tb03206.x

Nilsson-Ehle, H. (1936). Note regarding the gigas from of Populus tremula found in nature. Hereditas 21, 372-382.

Pécrix, Y., Géraldine, R., Hélène, F., Mireille, C., Serge, G., and Manuel, L. B. (2011). Polyploidization mechanisms: temperature environment can induce diploid gamete formation in Rosa sp. J. Exp. Bot. 62, 3587-3597. doi: 10.1093/ jxb/err052

Porch, T. G., and Jahn, M. (2001). Effects of high-temperature stress on microsporogenesis in heat-sensitive and heat-tolerant genotypes of Phaseolus vulgaris. Plant. Cell Environ. 24, 723-731. doi: 10.1046/j.1365-3040.2001.00716. $\mathrm{x}$

Ramanna, M. S., and Jacobsen, E. (2003). Relevance of sexual polyploidization for crop improvement-A review. Euphytica 133, 3-18.

Schuelke, M. (2000). An economic method for the fluorescent labeling of PCR fragments. Nat. Biotechnol. 18, 233-234. doi: 10.1038/72708 
Tian, J., Niu, X. Y., Tie, Y., and Lin, X. F. (2018). Meiosis of microsporocyte and morphology of pollen in hybrid of (Populus tomentosa $\times$ P. bolleana $) \times$ P. canescens. J. Northeast For. Univ. 46, 33-37. doi: 10.13759/j.cnki.dlxb.2018. 07.007

Tian, M., Zhang, Y., Liu, Y., Kang, X. Y., and Zhang, P. D. (2018). Pollen chromosome doubling induced by high temperature exposure to produce hybrid triploids in Populus canescens. Sci. Silva Sin. 54, 39-47. doi: 10.11707/ j.1001-7488.20180305

Veilleux, R. E. (1985). Diploid and polyploid gametes in crop plants: mechanisms of formation and utilization in plant breeding. Plant Breeding Rev. 3, 253-288. doi: 10.1002/9781118061008.ch6

Wang, J., Kang, X. Y., Li, D. L., and Chen, H. W. (2010a). Induction of diploid eggs with colchicine during embryo sac development in Populus. Silvae Genet. 59, 40-48. doi: 10.1515/sg-2010-0005

Wang, J., Kang, X. Y., Shi, L., Lu, M., and Yang, J. (2010b). Tetraploid induction of Populus hybrid in section Tacamahaca through zygotic chromosome doubling with physical and chemical treatments. J. Beijing For. Univ. 32, 63-66. doi: 10.13332/j.1000-1522.2010.05.020

Wang, J., Li, D. L., and Kang, X. Y. (2012). Induction of unreduced megaspores with high temperature during megasporogenesis in Populus. Ann. For. Sci. 69, 59-67. doi: 10.1007/s13595-011-0152-5

Xie, K. D., Xia, Q. M., Peng, J., Wu, X. M., Xie, Z. Z., Chen, C. L., et al. (2019). Mechanism underlying $2 \mathrm{n}$ male and female gamete formation in lemon via cytological and molecular marker analysis. Plant Biotechnol. Rep. 13, 141-149. doi: 10.1007/s11816-019-00525-4

Xu, C. P., Huang, Z., Liao, T., Li, Y., and Kang, X. Y. (2016). In vitro tetraploid plants regeneration from leaf explants of multiple genotypes in Populus. Plant Cell Tiss Org. 125, 1-9. doi: 10.1007/s11240-015-0922-0

$\mathrm{Xu}$, X. Q. (2008). Breeding of P. canescens. For.China 57.

Yang, J., Wang, J., Liu, Z., Xiong, T., Lan, J., Han, Q., et al. (2018). Megaspore chromosome doubling in Eucalyptus urophylla S.T. Blake induced by colchicine treatment to produce triploids. Forests 9:728. doi: 10.3390/f9110728

Yang, J., Yao, P., Li, Y., Mo, J. Y., Wang, J. Z., and Kang, X. Y. (2016). Induction of $2 \mathrm{n}$ pollen with colchicine during microsporogenesis in Eucalyptus. Euphytica 210, 69-78. doi: 10.1007/s10681-016-1699-x

Yang, Q., Chen, X., Zhang, P. D., and Kang, X. Y. (2015). Discrimination methods in $2 \mathrm{n}$ pollen in Populus. J. Northeast For. Univ. 43, 33-35. doi: 10.13759/j.cnki. dlxb.20141224.021

Zhang, H. L., Li, J. H., and Li, Z. Q. (2008). Studies on polyploid induction in vitro of Eucommia ulmoides Oliv. J. Northwest For. Univ. 23, 78-81. doi: 10.3724/SP. J.1005.2008.01033

Zhang, P. D., and Kang, X. Y. (2013). Occurrence and cytological mechanism of numerically unreduced pollen in diploid Populus euphratica. Silvae Genet. 62, 285-291. doi: 10.1515/sg-2013-0034
Zhang, P. D., Wu, F., and Kang, X. Y. (2012). Genotypic variation in wood properties and growth traits of triploid hybrid clones of Populus tomentosa at three clonal trials. Tree Genet. Genomes 8, 1041-1050. doi: 10.1007/s11295-0120484-9

Zhang, P. D., Wu, F., and Kang, X. Y. (2013). Genetic control of fiber properties and growth in triploid hybrid clones of Populus tomentosa. Scand. J. For. Res. 28, 621-630. doi: 10.1080/02827581.2013.829868

Zhang, P. D., Wu, F., and Kang, X. Y. (2015a). Chemical properties of wood are under stronger genetic control than growth traits in Populus tomentosa Carr. Ann. For. Sci. 72, 89-97. doi: 10.1007/s13595-014-0401-5

Zhang, P. D., Wu, F., Kang, X. Y., Zhao, C. G., and Li, Y. J. (2015b). Genotypic Variations of Biomass Feedstock Properties for Energy in Triploid Hybrid Clones of Populus tomentosa. BioEnerg Res. 8, 1705-1713. doi: 10.1007/s12155015-9622-1

Zhang, P. D., Wu, F., Kang, X. Y., and Tian, S. Y. (2014a). Genetic variation of fiber properties of triploid hybrid clones of white poplar. J. Northwest For. Univ. 29, 78-83. doi: 10.3969/j.issn.1001-7461.2014.01.16

Zhang, P. D., Wu, F., and Kang, X. Y. (2014b). Genetic variations in wood basic density and chemical components of triploid hybrid clones of white poplar. J. Northeast For. Univ. 42, 26-31. doi: 10.13759/j.cnki.dlxb.2014. 04.006

Zhao, C. G., Tian, M. D., Li, Y. J., and Zhang, P. D. (2017). Slow-growing pollentube of colchicine-induced $2 \mathrm{n}$ pollen responsible for low triploid production rate in Populus. Euphytica 213:94. doi: 10.1007/s10681-017-1881-9

Zhao, X. W., Peng, R. S., Ji, C. Y., and Liang, D. J. (2019). Inducing pollen chromosome doubling of Populus deltoid by colchicine. J. West China For. Sci. 48, 135-138. doi: 10.16473/j.cnki.xblykx1972.2019.01.023

Zhu, Z. T., Kang, X. Y., and Zhang, Z. Y. (1998). Studies on selection of natural triploids. Sci Silva Sin. 34, 22-31. doi: 10.3321/j.issn:1001-7488.1998. 04.004

Zhu, Z. T., Lin, H. B., and Kang, X. Y. (1995). Studies on allotriploid breeding of Populus tomentosa B301 clones. Sci. Silva Sin. 31, 499-505.

Conflict of Interest: The authors declare that the research was conducted in the absence of any commercial or financial relationships that could be construed as a potential conflict of interest.

Copyright (c) 2020 Zhou, Wu, Sang, Zhao, Zhang and Liu. This is an open-access article distributed under the terms of the Creative Commons Attribution License (CC BY). The use, distribution or reproduction in other forums is permitted, provided the original author(s) and the copyright owner(s) are credited and that the original publication in this journal is cited, in accordance with accepted academic practice. No use, distribution or reproduction is permitted which does not comply with these terms. 